\title{
Special Section on Selected Topics in Biophotonics: Optogenetics and Label-Free Optical Spectroscopy (Publisher's Note)
}




\section{Special Section on Selected Topics in Biophotonics: Optogenetics and Label-Free Optical Spectroscopy (Publisher's Note)}

[DOI: 10.1117/1.JBO.23.7.079801]

The three articles listed below were originally published in Volume 23, Issue 4 of the Journal of Biomedical Optics as part of the Special Section on Selected Topics in Biophotonics: Optogenetics and Label-Free Optical Spectroscopy. The special section was moved to a later issue after these articles were published, and as a result, the citation data has changed.

These articles now appear in the Journal of Biomedical Optics, Volume 23, Issue 7. They were republished online on 21 February 2018.

The corrected citations are shown below:

- Mikael Marois, Steven L. Jacques, Keith D. Paulsen, "Optimal wavelength selection for optical spectroscopy of hemoglobin and water within a simulated light-scattering tissue," Journal of Biomedical Optics 23(7), 071202 (26 January 2018).
- Jonas Golde, Florian Tetschke, Julia Walther, Tobias Rosenauer, Franz Hempel, Christian Hannig, Edmund Koch, Lars Kirsten, "Detection of carious lesions utilizing depolarization imaging by polarization sensitive optical coherence tomography," Journal of Biomedical Optics 23(7), 071203 (26 January 2018).

- Navchetan Awasthi, Sandeep Kumar Kalva, Manojit Pramanik, Phaneendra K. Yalavarthy, "Vector extrapolation methods for accelerating iterative reconstruction methods in limited-data photoacoustic tomography," Journal of Biomedical Optics 23(7), 071204 (5 February 2018). 